\title{
iPhone-Based Teleradiology for the Diagnosis of Acute Cervico-Dorsal Spine Trauma
}

\author{
Jayesh Modi, Pranshu Sharma, Alex Earl, Mark Simpson, J. Ross Mitchell, \\ Mayank Goyal
}

\begin{abstract}
Objective: To assess the feasibility of iPhone-based teleradiology as a potential solution for the diagnosis of acute cervico-dorsal spine trauma. Materials and Methods: We have developed a solution that allows visualization of images on the iPhone. Our system allows rapid, remote, secure, visualization of medical images without storing patient data on the iPhone. This retrospective study is comprised of cervico-dorsal computed tomogram (CT) scan examination of 75 consecutive patients having clinically suspected cervico-dorsal spine fracture. Two radiologists reviewed CT scan images on the iPhone. Computed tomogram spine scans were analyzed for vertebral body fracture and posterior elements fractures, any associated subluxation-dislocation and cord lesion. The total time taken from the launch of viewing application on the iPhone until interpretation was recorded. The results were compared with that of a diagnostic workstation monitor. Inter-rater agreement was assessed. Results: The sensitivity and accuracy of detecting vertebral body fractures was $80 \%$ and $97 \%$ by both readers using the iPhone system with a perfect inter-rater agreement (kappa:1). The sensitivity and accuracy of detecting posterior elements fracture was $75 \%$ and $98 \%$ for Reader 1 and $50 \%$ and $97 \%$ for Reader 2 using the iPhone. There was good inter-rater agreement (kappa: 0.66) between both readers. No statistically significant difference was noted between time on the workstation and the iPhone system. Conclusion: iPhone-based teleradiology system is accurate in the diagnosis of acute cervicodorsal spinal trauma. It allows rapid, remote, secure, visualization of medical images without storing patient data on the iPhone.
\end{abstract}

RÉSUMÉ: Téléradiologie basée sur le iPhone pour le diagnostic du traumatisme aigu de la colonne cervicale et dorsale. Objectif : Le but de l'étude était d'évaluer la faisabilité de la téléradiologie basée sur le iPhone comme ressource pour le diagnostic du traumatisme de la colonne cervicale et dorsale.Méthodologie : Nous avons développé une ressource qui permet la visualisation d'images sur le iPhone. Notre système permet la visualisation rapide, à distance et sécurisée d'images médicales sans sauvegarder les informations concernant le patient dans le iPhone. Cette étude rétrospective inclut l'examen de tomodensitométrie cervico-dorsale de 75 patients consécutifs chez qui on soupçonnait cliniquement une fracture de la colonne cervico-dorsale. Deux radiologistes ont révisé les tomodensitométries sur le iPhone pour détecter les fractures des corps vertébraux et des éléments postérieurs, ainsi que les subluxations-dislocations et les lésions de la moelle épinière associées. Le temps total à partir du lancement de l'application sur le iPhone jusqu'à l'interprétation a été noté. Les résultats ont été comparés à ceux obtenus à un poste de travail utilisé pour le diagnostic et la concordance entre les observateurs a été évaluée. Résultats : La sensibilité et l'exactitude de détection des fractures du corps vertébral étaient de $80 \%$ et $97 \%$ respectivement pour les deux observateurs lorsqu'ils utilisaient le système iPhone, et la concordance inter-observateurs était parfaite (kappa : 1). La sensibilité et l'exactitude de détection pour les fractures des éléments postérieurs étaient de 75\% et 98\% respectivement pour l'observateur 1 et de $50 \%$ et $97 \%$ pour l'observateur 2 sur le iPhone. La concordance inter-observateurs était bonne (kappa : 0,66) entre les deux observateurs. Aucune différence significative au point de vue statistique n'a été notée quant au temps consacré au poste de travail et au système iPhone. Conclusion : Le système de téléradiologie basé sur le iPhone pour le diagnostic du traumatisme aigu de la colonne cervico-dorsale est exact. Il permet une visualisation rapide, à distance et sécurisée des images médicales sans sauvegarde des données du patient dans le iPhone.

Can. J. Neurol. Sci. 2010; 37: 849-854

An instant image transfer system can be used to support rapid medical and imaging decision making in an emergency situations when doctors are away from the hospital ${ }^{1-3}$. There are several Web-based picture archiving and communication systems, which allows access to a patient's radiological images from outside the hospital ${ }^{1}$. However, they are felt to be not appropriate for use in time-critical emergency cases as mentioned in previous studies ${ }^{4,5}$.

Handheld devices like smart-phones and personal digital assistants (PDAs) have become increasingly prevalent in modern society $^{6-8}$ and $46 \%$ of attending physicians and trainees ${ }^{9}$ and $45 \%$ of radiologists are using handheld devices ${ }^{8-10}$. However, there are several limitations: small screen size, inherent low resolution of display, low contrast ratio, poor connectivity, slow data transfer, security issues, and minimal inherent memory ${ }^{8,11}$. Methods for image transfer using commonly available PDAs

From the Department of Radiology (JM, PS, AE, MS, JRM, MG), Department of Clinical Neurosciences (JRM, MG), Foothills Medical Centre, University of Calgary, Calgary, Alberta, Canada.

Received April 27, 2010. Final Revisions Submitted June 11, 2010. Correspondence to: Mayank Goyal, Radiology and Clinical Neurosciences,

Department of Radiology, Foothills Medical Centre, University of Calgary, 1403, 29th St. NW, Calgary, Alberta, T2N 2T9, Canada. 
have been addressed by many investigator ${ }^{1-3,12,13}$. However, patient confidentiality, especially when medical information is downloaded on a portable device, remains a significant concern.

We have developed a new teleradiology system based on a client/server architecture to address the limitations related to connectivity and confidentiality. Our system can use an Apple iPhone or iPod Touch (hereafter referred to as iPhone system) as a remote visualization device. Our solution allows rapid, remote, secure, two-dimensional (2D) and three-dimensional (3D) visualization of medical images on an iPhone device wherever wireless network connectivity is available. Additionally, our solution does not require patient data to be stored on the iPhone. This allows immediate visualization of new patient images from remote locations, an important feature when timely interpretation is crucial for appropriate treatment.

Acute spinal trauma is commonly encountered in emergency radiology, and accurate and timely diagnosis is important. Here we have assessed the feasibility of using an iPhone teleradiology system for the instant transmission of radiological images and its usefulness for making an effective medical decision in emergency conditions like in acute cervico-dorsal spinal trauma patients.

\section{Materials And Methods}

This retrospective study is comprised of cervico-dorsal computed tomogram (CT) scans examination of 75 consecutive patients with clinically suspected cervico-dorsal spinal fracture. An ethics application was made to the Institutional Review Board. In their opinion, the project was felt to be program evaluation, quality assurance and quality improvement work and did not require a formal approval from the Ethics Board.

\section{CT Cervico-dorsal spine evaluation}

Computed tomogram scans were performed on the 64-rowmultidetector CT (Somatom Sensation 64, Siemens Medical Solutions, Germany). Scan coverage was from the base of skull to the T5 vertebral body level using helical acquisition and a bone algorithm. Slices were obtained at a slice thickness/interval of $2 / 1 \mathrm{~mm}, \mathrm{KV} / \mathrm{mAS} /$ collimation of $140 / 300 / 64 \times 0.6$ and a pitch/rotation time of $0.9 / 1 \mathrm{sec}$. Reformations with $1 \mathrm{~mm}$ slices were performed in the axial, saggital and coronal planes.
Reader 1 (PS) first interpreted the CT scans on a radiology workstation and after a 4-week interval, again viewed them on an iPhone. The workstation (IMPAX 6.3.1.3815, Agfa Healthcare, Belgium) was connected to a medical-grade 21-inch liquid crystal display (MD21GS-3MP, NEC). This display has a resolution of $2048 \times 1536$ pixels (pixel pitch $=0.21 \mathrm{~mm}$ ), 10-bit grayscale depth display and a calibrated luminance of $400 \mathrm{~cd} / \mathrm{m}^{2}$.

Reader 2 (JM) interpreted images only on the iPhone. A tutorial on how to use the iPhone system was provided to the readers. The patient exams were presented in different orders on the iPhone teleradiology system to reduce any recall bias. The CT spine scans were analyzed for vertebral body fracture and posterior elements fractures, any associated subluxationdislocation and cord lesion. In this study, all patient imaging exams were anonymized and uploaded on the visualization server running client software (ResolutionMD Enterprise).

Communication between the visualization server and the iPhone device occurred over a secure wireless network (Wi-Fi 802.11g). The user initially launches the viewing application (ResolutionMD mobile software) on the iPhone. On establishing communication with the visualization server, CT scan study file is selected to visualize on the iPhone. We recorded total time taken from the launch of client viewing application on the iPhone followed by selection of CT scan study file of the patient until the interpretation was complete. The time taken from the launch of viewing application until the display of the first image on the iPhone was not measured separately, but rather was included in the total time. The results were compared with that of a diagnostic workstation monitor. Inter-rater agreement was assessed.

\section{The iPhone-based teleradiology system}

The teleradiology system included a visualization server and an iPhone/iPod Touch (Apple Inc., Cupertino, CA). The iPod Touch and iPhone $3 \mathrm{G}$ have identical displays. Both devices run the same iPhone Operating System. The major difference is that the iPod Touch lacks cellular telephone and global positioning system circuitry. The iPhone/iPod Touch included $8 \mathrm{~GB}$ of flash memory and 3.5-inch liquid crystal display diagonal screen with $320 \times 480$ pixels (pixel pitch $=0.15 \mathrm{~mm}$ ), 2:3 aspect ratio, 8-bit grayscale depth display and a luminance of $500 \mathrm{~cd} / \mathrm{m} 2$. It ran a

Table 1: Positive predictive value, negative predictive value, sensitivity and accuracy of acute cervico-dorsal spine trauma diagnosis using the iPhone-based teleradiology system by Reader 1 (R1)

\begin{tabular}{|c|c|c|c|c|c|c|c|c|c|c|}
\hline & $\begin{array}{l}\text { Work- } \\
\text { station }\end{array}$ & $\begin{array}{l}\text { iPhone } \\
\text { (R1) }\end{array}$ & $\mathrm{TP}$ & FP & $\mathrm{TN}$ & $\mathrm{FN}$ & PPV & NPV & Sensitivity & Accuracy \\
\hline $\begin{array}{l}\text { Vertebral body } \\
\text { fractures }\end{array}$ & $5 / 66$ & $6 / 66$ & 4 & 1 & 60 & 1 & $80 \%$ & $98 \%$ & $80 \%$ & $97 \%$ \\
\hline $\begin{array}{l}\text { Posterior elements } \\
\text { fractures }\end{array}$ & $4 / 66$ & $4 / 66$ & 3 & 0 & 62 & 1 & $100 \%$ & $98 \%$ & $75 \%$ & $98 \%$ \\
\hline
\end{tabular}

$\mathrm{n}=$ Total number of patients having fracture detected on workstation; $\mathrm{R} 1=$ Total number of patients having fracture detected on iPhone by Reader 1; R2 = Total number of patients having fracture detected on iPhone by Reader 2; TP=True positive on the iPhone device; FP=False positive on the iPhone device; $\mathrm{TN}=$ True negative on the iPhone device; FN= False negative on the iPhone device; $\mathrm{PPV}=$ Positive predictive value; $\mathrm{NPV}=$ Negative predictive value 
client program ResolutionMD Mobile (Calgary Scientific Inc., Calgary, Canada) that handled user input, communication with the visualization server, and the display of transmitted images. The client software allows $2 \mathrm{D}$ and $3 \mathrm{D}$ visualization with interactive window/level, translation, rotation, and zoom capabilities. In 3D mode the user can also select from a range of tissue rendering modes.

The visualization server had a $2.4 \mathrm{GHz}$ Intel Core 2 Quad central processing unit, 8 GB RAM and 2 nVidia GeForce 8800 (512 MB) graphics cards. It ran the application ResolutionMD Enterprise (Calgary Scientific Inc., Calgary, Canada), which allows remote $2 \mathrm{D} / 3 \mathrm{D}$ visualization of digital imaging and communications in medicine (DICOM) images through a webbrowser on an iPhone device. Visualization server software decomposes the sequence of $2 \mathrm{D}$ frames into static and dynamic components and reformats them into a $3 \mathrm{D}$ volume. It then performs a rendering operation on the $3 \mathrm{D}$ volume to produce $2 \mathrm{D}$ "frame", which is independently compressed using the joint photographic experts group algorithm and encoded for transmission to the remote client for display on the iPhone. The decomposition and compression algorithms can adapt to the achievable bandwidth between the server and the client. During user interaction, only the dynamic components of the sequence are transmitted, and a lossy compression algorithm is used. This reduces the amount of data that must be transmitted to the client to update the display. The level of lossy compression can be adjusted by the user from the client program. Higher compression levels allow a faster frame rate. When the user stops interacting with the server rendering system, a lossless frame is automatically transmitted to the remote client. This allows higher frame rates (temporal resolution) during interaction, but full quality still images (spatial resolution) during static viewing.

Communication between the visualization server and the iPhone device occurred over a secure wireless network (Wi-Fi $802.11 \mathrm{~g})$. This system is capable of delivering and displaying up to 14 images per second on the iPhone $3 \mathrm{GS}$. In our experience, a single visualization server can accommodate ten or more simultaneous iPhone device users. Importantly, all patient data remains on the visualization server with this system. The streams of rendered images are not saved on the iPhone device. When the iPhone device client software is terminated, the network connection to the server is automatically closed. This system design has several important features. First, confidential patient information is not stored on the iPhone device and it could potentially be carried outside the hospital. Second, the visualization server can rapidly load, and render, large medical image datasets containing several hundred DICOM images. This allows diagnostic interpretation to begin almost immediately, even from remote locations.

\section{Statistical Analysis}

The workstation results were considered the standard against which the readings on the iPhone device were compared. Any difference in detecting number of vertebral body or posterior elements fracture/s in a patient on the iPhone system as compared to workstation was considered a discrepancy. True positives, true negatives, false positives and false negatives were obtained. The positive predictive value, negative predictive value, sensitivity and accuracy were calculated. Inter-rater agreement was measured by calculation of Cohen's Kappa using Stata 10.0 (StataCorp, College Station, Texas, USA). The total time taken from the launch of viewing application on the iPhone until interpretation was recorded and compared to that of a workstation.

\section{RESULTS}

Cervico-dorsal CT scans of 75 consecutive patients with clinically suspected cervico-dorsal spinal fracture were obtained from our radiology database. Poor image quality resulted in nine patients excluded from our study, resulting 66 patients CT scan exams for review. A total of six vertebral body fractures were diagnosed in 5/66 patients on the workstation. There were two C2, two T3 and two T5 vertebral body fractures. The sensitivity of detecting vertebral body fractures on iPhone was $80 \%$ and the accuracy was $97 \%$ by both the readers (Table 1 and 2). Both readers detected fractures correctly in $4 / 5$ patients on the iPhone device (Figure 1). However both readers had one false positive and one false negative diagnosis on the iPhone device. Both readers falsely diagnosed one patient having a T3 vertebral body fracture thus having one false positive diagnosis on the iPhone. One patient had fracture involving the $\mathrm{C} 2$ and the $\mathrm{T} 5$ vertebral

Table 2: Positive predictive value, negative predictive value, sensitivity and accuracy of acute cervico-dorsal spine trauma diagnosis using the iPhone-based teleradiology system by Reader 2 (R2)

\begin{tabular}{|c|c|c|c|c|c|c|c|c|c|c|}
\hline & $\begin{array}{l}\text { Work- } \\
\text { station }\end{array}$ & $\begin{array}{l}\text { iPhone } \\
\text { (R2) }\end{array}$ & $\mathrm{TP}$ & FP & $\mathrm{TN}$ & $\mathrm{FN}$ & PPV & NPV & Sensitivity & Accuracy \\
\hline $\begin{array}{l}\text { Vertebral body } \\
\text { fractures }\end{array}$ & $5 / 66$ & $6 / 66$ & 4 & 1 & 60 & 1 & $80 \%$ & $98 \%$ & $80 \%$ & $97 \%$ \\
\hline $\begin{array}{l}\text { Posterior elements } \\
\text { fractures }\end{array}$ & $4 / 66$ & $4 / 66$ & 2 & 0 & 62 & 2 & $100 \%$ & $97 \%$ & $50 \%$ & $97 \%$ \\
\hline
\end{tabular}

$\mathrm{n}=$ Total number of patients having fracture detected on workstation; $\mathrm{R} 1=$ Total number of patients having fracture detected on iPhone by Reader 1; R2= Total number of patients having fracture detected on iPhone by Reader 2; TP=True positive on the iPhone device; $\mathrm{FP}=$ False positive on the iPhone device; $\mathrm{TN}=$ True negative on the iPhone device; FN= False negative on the iPhone device; $P P V=$ Positive predictive value; NPV= Negative predictive value 

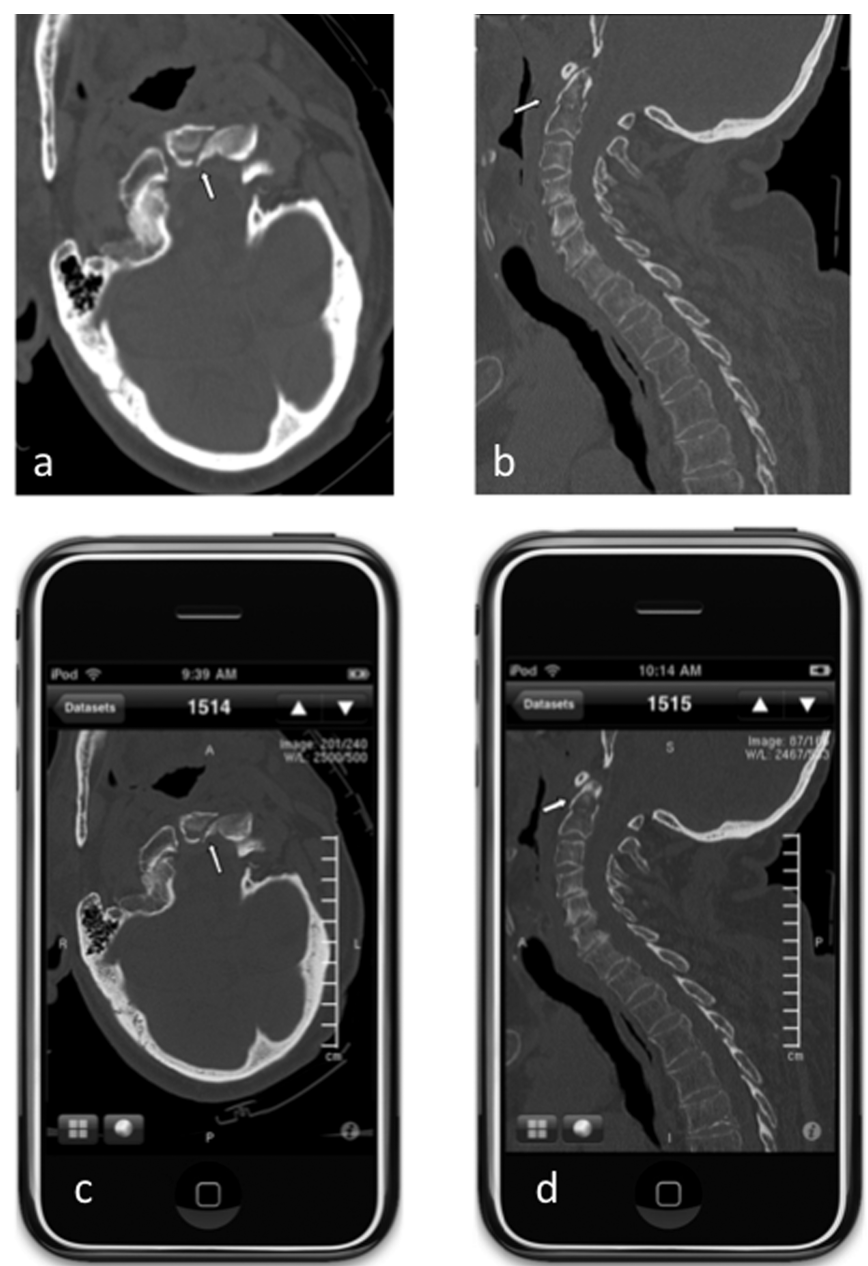

Figure 1: A 54-year-old male presented with acute spinal trauma. Axial and saggital views of the CT spine cervico-dorsal region shows fracture of the dens on the workstation images $(a, b)$. Corresponding images on the iPhone device were interpreted accurately $(c, d)$.

body. Both readers correctly diagnosed a $\mathrm{C} 2$ fracture, but missed a T5 subtle fracture leading to one false negative diagnosis. One patient had associated dislocation, which was correctly diagnosed on iPhone system by both the readers. None of the patients had any cord compression or injury. Both the readers had perfect inter-rater agreement (kappa: 1) for diagnosing vertebral body fracture on the iPhone system.

Any fracture in pedicle, lamina, spinous and transverse process were considered posterior elements fracture. A total of 4/66 patients with posterior elements fractures were detected on the workstation. There were one pedicle, five spinous processes and one transverse process fracture. The sensitivity of detecting posterior elements fracture on the iPhone was $75 \%$ and $50 \%$ by Reader 1 and 2 respectively. The accuracy of detecting posterior elements fracture on the iPhone was $98 \%$ and $97 \%$ by Reader 1 and 2 respectively. There were no false positives. Reader 1 had one false negative and Reader 2 had two false negatives on the iPhone device. One patient had C3, C4, T1 and T2 spinous process fracture. Both readers correctly diagnosed $\mathrm{C} 4$, $\mathrm{T} 1$ and $\mathrm{T} 2$ spinous process fracture (Figure 2). However, both readers missed a C3 spinous process fracture on the iPhone. Reader 2 had also missed one C5 spinous process fracture in one patient. Both the readers had good inter-rater agreement (kappa: 0.66) for diagnosing posterior element fracture on the iPhone system.

The average time to interpret cervico-dorsal spine CT scan on the workstation was $6.8 \pm 2.6$ minutes (mean $\pm \mathrm{SD}$ ), while using the iPhone system was $6.7 \pm 2.1$ minutes by Reader 1 and $6.8 \pm 2.3$ minutes by Reader 2 . No statistically significant difference was noted in the time to interpret scans on the workstation and the iPhone system.

\section{DISCUSSION}

Handheld devices like smartphones and PDAs may be comparable with secondary-display monitors used in emergency teleconsultation for reporting findings on acute intracranial hemorrhage on CT images and fractured wrists on radiographs ${ }^{8}$. There are now many studies which have outlined the potential
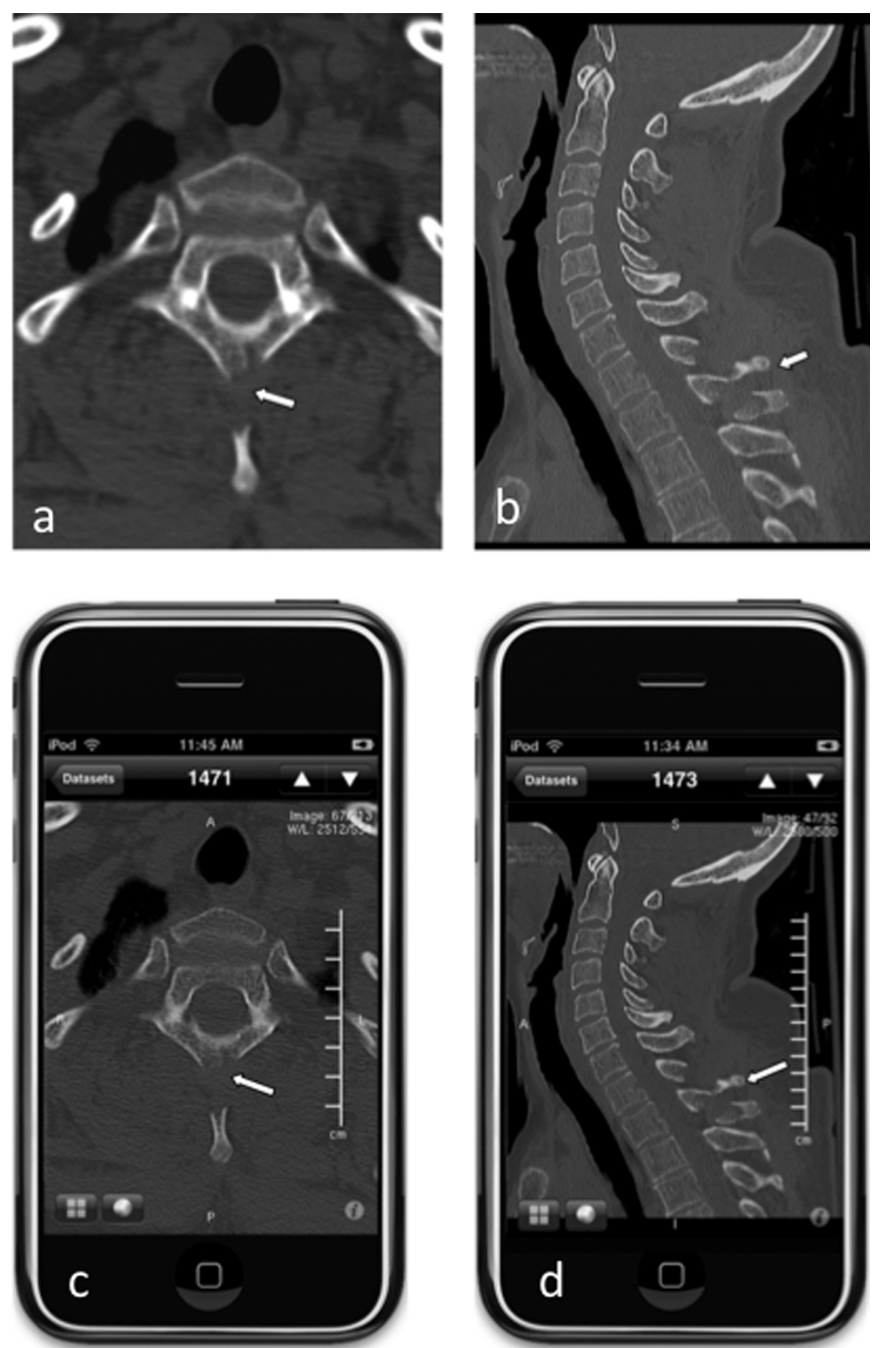

Figure 2: A 39-year-old male presented with acute spinal trauma. A CT spine of cervico-dorsal region shows fracture of C4, T1, T2 spinous process in workstation image $(a, b)$. Corresponding images on the iPhone device were interpreted accurately $(c, d)$. 
usefulness of handheld devices for the evaluation of emergency CT scans ${ }^{3,12,14}$, renal colic ${ }^{15}$, intracranial hemorrhage ${ }^{4}$, appendicular skeletal trauma (Chew NS et al, presented at the 2008 annual meeting of the Radiological Society of North America) and dental images ${ }^{16}$.

Injury to spine is one of the most feared traumas, and spinal cord injury is a major cause of disability. Since most of the spinal trauma patients survive their injuries, most of them end with partial or complete paralysis. It requires accurate and emergent imaging assessment of in order to evaluate spinal stability and integrity of neural elements. A number of studies have advocated early reduction (four to ten hours) and operative fixation of spinal fractures in patients with acute spinal cord injury ${ }^{17-20}$. Computed tomography is the preferred initial imaging modality in acute spine trauma patients. Not only is CT more accurate in diagnosing spinal injury, it also reduces imaging time and patient manipulation $^{21}$.

Here, we have assessed the feasibility of using iPhone teleradiology system for the instant transmission of radiological images in acute cervico-dorsal spinal trauma patients, and its usefulness for making an effective medical decision in an emergency. In this study, the sensitivity of detecting vertebral body fractures on iPhone was $80 \%$ and the accuracy rate was $97 \%$ by both the readers. There was one false positive and one false negative in detecting vertebral body fracture by both the readers. One patient was misdiagnosed as having a T3 vertebral body fracture, which was confirmed, on a workstation as detached osteophyte fragment. One patient had a C2 and T5 vertebral body fracture. The $\mathrm{C} 2$ fracture was correctly diagnosed but subtle fracture in the antero-superior aspect of T5 vertebral body was missed.

The sensitivity of detecting posterior elements fracture on the iPhone was $75 \%$ and $50 \%$ by Reader 1 and 2 respectively. The accuracy rate of detecting posterior element fractures on the iPhone was $98 \%$ by Reader 1 and $97 \%$ by Reader 2 . There was one false negative by Reader 1 and two false negative by Reader 2. One patient had a fracture involving the spinous process of the C3, C4, T1 and T2 vertebrae. Both readers correctly diagnosed $\mathrm{C} 4, \mathrm{~T} 1$ and $\mathrm{T} 2$ spinous process fracture, but missed subtle $\mathrm{C} 3$ spinous process fracture. Reader 2 had one more false negative due to missing one $\mathrm{C} 5$ spinous process fracture. We felt these false diagnoses were due to misinterpretation rather than poor image quality or display. None of the missed fractures had evidence of spinal canal compromise or presence of features that would suggest an unstable fracture. None of these false diagnoses would have changed patient management. We had comparable high accuracy rates on the iPhone system though the display is much smaller than that of a clinical workstation. We found that interactive zoom of the CT images allowed users to easily focus on regions of interest. When interpreting images, users tend to position the iPhone display much closer to their eyes, which helped compensate, for the smaller size of iPhone display. We found factors in favor of the iPhone display are its pixel density and luminance. Both were $25 \%$ higher than those on the medical grade display used in these experiment. Even though interpretations occurred under normal office lighting conditions, the subjective opinion of the radiologists was that the iPhone display was high quality, provided accurate gray scale representations of the image data, and was sufficient for this diagnostic task. The iPhone ran a client program that allows $2 \mathrm{D}$ and 3D visualization with interactive window/level, rotation and zoom capabilities. The iPhone client software used in this study was not having multiplanar reconstruction application. However, new software does provide functions for multi-planar reformatting. We are investigating methods to usefully expose additional advanced visualization capabilities like measuring density (Hounsfield units) to the user on the iPhone device.

Our results reflects that of Ryan et al (Proceedings from UK Radiological congress 2008) who found a personal digital assistant to be accurate in diagnosing acute conditions like detecting intracranial hemorrhage when comparing it with a workstation. Ryan et al converted the images to bitmap format and transferred them to a PDA. It is not clear in the study that the images were then on the hard drive of the PDA. Also, the authors did not discuss potential patient confidentiality issues. Loss or theft of a PDA is not rare. As such, we feel that solutions based downloading images to the hard drive of the device may not be safe. Our solution is Internet based and doesn't require patient data to be stored on the iPhone. Hence, patient confidentiality is not compromised in case of loss or theft.

Today, a physician on call must travel to a workstation, potentially power it on and wait for it to start-up, then login and launch the viewing application before interpretation can begin. If the physician is located outside their hospital or home, travel time may be significant. By comparison, our iPhone device/ visualization server teleradiology system is always-on, takes only a few seconds to securely access remote imaging exams anywhere cellular or wireless network is available and can be carried conveniently by a physician while on call. The average time to interpret cervico-dorsal spine CT scan on the workstation was $6.8 \pm 2.6$ minutes $($ mean $\pm \mathrm{SD})$, while using the iPhone system was $6.7 \pm 2.1$ minutes by Reader 1 and $6.8 \pm 2.3$ minutes by Reader 2 . No statistically significant difference was noted in the time to interpret scans on workstation and iPhone device. Thus it can allow rapid visualization of radiological images and urgent management decisions. If the Wi-Fi network is poor quality, the frame rate could also be low. However, in our experience outside the laboratory, frame rate has been acceptable (10 frames per second or more) over the less than perfect Wi-Fi connections. The performance can be fine even if the distance between the server and iPhone is several kilometers. Our iPhone Client Server can accommodate ten or more simultaneous iPhone device users, and is capable of delivering and displaying up to 14 frames per second on an iPhone device connected over an 802.11g Wi-Fi network. This frame rate provided sufficient interactivity for comfortable use. However, the frame rate of our system using a $3 \mathrm{G}$ cellular network was much lower, typically between one and four frames per second. This was deemed by the radiologists to be insufficient for practical use. The $4 \mathrm{G}$ cellular networks have been, or are being, installed in most metropolitan centers in North America, Europe and parts of Asia, which may be sufficient for practical use. We estimate that the higher bandwidth of these new cellular networks should allow 10-15 frames per second to be delivered to an iPhone. However, the current iPhone $3 \mathrm{GS}$ is not able to use $4 \mathrm{G}$ networks. An updated iPhone device will also be required.

Our study has limitations. It is a retrospective analysis performed on only a small number of patients by two radiologists, who were located in a research laboratory environment, with robust, high-speed network infrastructure. 
Since this study was proof of principle to study the feasibility of use of iPhone-based teleradiology for the diagnosis of acute cervico-dorsal spine trauma, we considered diagnosis made by only one radiologist as the standard for comparison. However, in future prospective studies, we would like to measure inter-rater as well as intra-rater agreement on the iPhone and the workstation. A larger prospective study, performed by physicians on-call outside the laboratory, operating under clinical constraints and clinical deadlines, will be required to demonstrate clinical utility of our new system. Also, to be truly effective, the system should provide practical frame rates over cellular networks. This would greatly extend the area of network coverage, and system utility.

\section{CONClusions}

In summary, the iPhone-based teleradiology system is accurate in the diagnosis of acute cervico-dorsal spinal trauma. Though the iPhone system is unlikely to replace a workstation for definitive report, it has the potential to allow urgent management decisions where time is important factor. It allows rapid visualization of large imaging datasets and manages concerns related to patient confidentiality. This system might also be useful in other acute care scenarios, such as the evaluation of emergency CT scans, renal colic, acute ischemic stroke, and appendicular skeletal trauma, where rapid access to image data is an important factor determining patient outcome. The system also might be useful as a bedside teaching tool to help physicians communicate better with patients and their families.

Our future efforts will be focused on addressing some of limitations of the current study. In particular, we are investigating methods to usefully expose additional advanced visualization capabilities like measuring density (Hounsfield units) to the remote user on the iPhone device. We are conducting additional prospective studies to evaluate potential clinical utility in more detail. We are investigating new methods to optimize both server and client performance to enhance interactivity over both $\mathrm{Wi}-\mathrm{Fi}$ and cellular networks.

\section{CONFLICTS OF INTEREST}

J. Ross Mitchell is the founder and chief scientist of Calgary Scientific Inc (CSI). Mayank Goyal and J. Ross Mitchell are shareholders of CSI. The iPhone client software was originally developed by J. Ross Mitchell and Mark Simpson as a research platform. The source code was subsequently transferred to CSI. No equity in CSI was involved in this exchange. Calgary Scientific Inc enhanced and improved the source code to create ResolutionMD Mobile. The value of CSI shares may increase if ResolutionMD Mobile becomes a successful product.

\section{ACKNOWLEDGMENT}

J. Ross Mitchell is funded by the Alberta Informatics Circle of Research Excellence and by the Alberta Heritage Foundation for Medical Research.

\section{REFERENCES}

1. Kim DK, Yoo SK, Park JJ, Kim SH. PDA-phone-based instant transmission of radiological images over a CDMA network by combining the PACS screen with a Bluetooth-interfaced local wireless link. J Digit Imaging. 2007 Jun;20(2):131-9.

2. Yamada M, Watarai H, Andou T, Sakai N. Emergency image transfer system through a mobile telephone in Japan: technical note. Neurosurgery. 2003 Apr;52(4):986-8; discussion 8-90.

3. Yamamoto LG, Williams DR. A demonstration of instant pocket wireless CT teleradiology to facilitate stat neurosurgical consultation and future telemedicine implications. Am J Emerg Med. 2000 Jul;18(4):423-6.

4. Yaghmai V, Kuppuswami S, Berlin JW, Salehi SA. Evaluation of personal digital assistants as an interpretation medium for computed tomography of patients with intracranial injury. Emerg Radiol. 2003 Oct;10(2):87-9.

5. Yaghmai V, Salehi SA, Kuppuswami S, Berlin JW. Rapid wireless transmission of head CT images to a personal digital assistant for remote consultation. Acad Radiol. 2004 Nov;11(11):1291-3.

6. Fischer S, Stewart TE, Mehta S, Wax R, Lapinsky SE. Handheld computing in medicine. J Am Med Inform Assoc. 2003 MarApr;10(2):139-49.

7. Ebell M, Rovner D. Information in the palm of your hand. J Fam Pract. 2000 Mar;49(3):243-51.

8. Toomey RJ, Ryan JT, McEntee MF, Evanoff MG, Chakraborty DP, McNulty JP, et al. Diagnostic efficacy of handheld devices for emergency radiologic consultation. AJR Am J Roentgenol. 2010 Feb;194(2):469-74.

9. McLeod TG, Ebbert JO, Lymp JF. Survey assessment of personal digital assistant use among trainees and attending physicians. $\mathbf{J}$ Am Med Inform Assoc. 2003 Nov-Dec;10(6):605-7.

10. Boonn WW, Flanders AE. Informatics in radiology (infoRAD): survey of personal digital assistant use in radiology. Radiographics. 2005 Mar-Apr;25(2):537-41.

11. Flanders AE, Wiggins RH, 3rd, Gozum ME. Handheld computers in radiology. Radiographics. 2003 Jul-Aug;23(4):1035-47.

12. Reponen J, Ilkko E, Jyrkinen L, Tervonen O, Niinimaki J, Karhula $\mathrm{V}$, et al. Initial experience with a wireless personal digital assistant as a teleradiology terminal for reporting emergency computerized tomography scans. J Telemed Telecare. 2000;6(1): 45-9.

13. Raman B, Raman R, Raman L, Beaulieu CF. Radiology on handheld devices: image display, manipulation, and PACS integration issues. Radiographics. 2004 Jan-Feb;24(1):299-310.

14. Kondo Y. [Medical image transfer for emergency care utilizing internet and mobile phone]. Nippon Hoshasen Gijutsu Gakkai Zasshi. 2002 Oct;58(10):1393-401.

15. Johnston WK, 3rd, Patel BN, Low RK, Das S. Wireless teleradiology for renal colic and renal trauma. J Endourol. 2005 Jan-Feb;19(1):32-6.

16. Salo S, Salo H, Liisanantti A, Reponen J. Data transmission in dental identification of mass disaster victims. J Forensic Odontostomatol. 2007 Jun;25(1):17-22.

17. Aebi M, Mohler J, Zach GA, Morscher E. Indication, surgical technique, and results of 100 surgically-treated fractures and fracture-dislocations of the cervical spine. Clin Orthop Relat Res. 1986 Feb(203):244-57.

18. Wiberg J, Hauge HN. Neurological outcome after surgery for thoracic and lumbar spine injuries. Acta Neurochir (Wien). 1988;91(3-4):106-12.

19. Wolf A, Levi L, Mirvis S, Ragheb J, Huhn S, Rigamonti D, et al. Operative management of bilateral facet dislocation. J Neurosurg. 1991 Dec;75(6):883-90.

20. Hadley MN, Fitzpatrick BC, Sonntag VK, Browner CM. Facet fracture-dislocation injuries of the cervical spine. Neurosurgery. 1992 May;30(5):661-6.

21. Van Goethem JW, Maes M, Ozsarlak O, van den Hauwe L, Parizel PM. Imaging in spinal trauma. Eur Radiol. 2005 Mar;15(3): 582-90. 